\title{
Support of the upper limbs of office workers during a daily work journey
}

\author{
Ernesto Filgueiras ${ }^{\mathrm{a}, \mathrm{b}, *}$, Francisco Rebelo ${ }^{\mathrm{b}}$ and Moreira da Silva ${ }^{\mathrm{c}}$ \\ ${ }^{a}$ Beira Interior University - R. Marquês d'Ávila e Bolama, 6201-001 Covilhã - Portugal \\ ${ }^{b}$ CIPER - "Interdisciplinary Centre for the Study of Human Performance" - Ergonomics Laboratory. FMH / Technical \\ University of Lisbon, Estrada da Costa, 1499-002 Cruz Quebrada - Dafundo, Portugal \\ ${ }^{c}$ Faculty of Architecture - Technical University of Lisbon - R. Sá Nogueira, Alto da Ajuda 1349-055 Lisbon, Portugal
}

\begin{abstract}
Generally, the studies assess the upper limbs postures during the work with Visual Display Terminal - VDT through the measurement of the angles formed by the segments or through the relation with the pre-defined anatomical postures (e.g., flexion, extension, abduction). However, few studies were found in the literature which had focused in the measurement of the upper limbs support during a real situation of work with VDT and in the definition of analysis categories for this kind of behavior. The main objective of this study was to measure, in a real situation and using a systematic observation method through video analysis, the main kinds of support for the upper limbs. The analysis was done considering 480 work hours of 30 office workers. Data were collected using a methodology proposed by Rebelo, Filgueiras \& Soares [1] and related with the work organization and workspace conditions, the participants had: a) minimum of eight hours daily of work; b) similar activities, characterized as office work; c) same furniture and equipment; and, d) computers with the same hardware, software and peripherals. Results were analyzed 46554 dynamic events, considering only the fourteen Interaction Categories - ICs, for upper limps support. In this way, percentage was computed, considering the total number of occurrence, for the Right arm is: arm support $(0.06 \%)$; forearm support $(31,8 \%)$; elbow support $(31,92 \%)$; hand support $(7,41 \%)$; multiple supports $(12,32 \%)$ and without support $16,41 \%$ - For the Left arm the results is: forearm support $(0,11 \%)$; arm support $(53,69 \%)$; elbow support $(8,70 \%)$; hand support $(7,97 \%)$; multiple supports $(8,43 \%)$ e without support $(21,11 \%)$. This systemic and ecological approach was obtained through a method which enables experimental Biomechanics and Physiology methods to develop more efficient functional requirements and recommendations for the work with VDTs.
\end{abstract}

Keywords: Upper limbs support, Human interaction, Systematic observation of work in a systemic and ecological approach, Ergonomics methods of analysis, Video-analysis technics

\section{Introduction}

The importance of body supports during the work, namely in works with Visual Display Terminals VDT, is recognized in the literature as one of the most efficient strategies to combat physical fatigue and the Cumulative Trauma Disorders - CTDs [2-4].

Likewise the lack of support for the upper limbs is a major concern of designers during the development of equipment.[5].

Most of the studies that assess the work activity with VDT focused in the analysis of the upper limbs posture. Additionally, it is very difficult to find in the literature information about the place where the workers their upper limbs. However, knowing how this support is done, what regions are predominantly supported and how many times the armrest of the chair is used (as it is an element which is required for approval of a chair as an "office chair" and has a financial cost) and is an important issue.

To measure the interaction in office situation has been a concern among the researchers on the Ergonomics area. The self-reports, which are often used in this context [6] were very contested by Spielholz and colleagues [7]. These authors considered the selfreport to be less reliable when compared to direct methods of data collection or observation. The methods based on direct data collection rely on instru-

\footnotetext{
Corresponding authors. E-mail: ernestovf@gmail.com / frebelo@fmh.utl.pt / fms@gmail.com .
} 
ments which have often high financial costs and limitations on data collection during a working day. The systematic observation has been presented as an effective method to collect information about the postural behavior during long periods of time [8].

The existence of supports for the body segments during the the seated work is one of the most important and effective strategies to combat the physical fatigue and the occurrence of Repetitive Strain Injury - RSI [2, 9].

In this context, this paper aims to present a study which the main objective is to measure, in a real work situation and using a systematic observation method through video analysis (Behavior Video), the main kinds of support for the upper limbs. The analysis was done considering 480 work hours of 30 office workers.

\section{Methodology}

Data were collected using a methodology proposed by Rebelo, Filgueiras \& Soares [1]. Thirty workers (mean age $=32$ years old, $\mathrm{SD}=8$ ) of an office of a service company participated in this study. The participants declared through interview having the right hand as dominant. Related with the work organization and workspace conditions, the participants had: a) minimum of eight hours daily of work; b) similar activities, characterized as office work; c) same furniture and equipment; and, d) computers with the same hardware, software and peripherals.

\subsection{Phase I-Analysis of Reference Situation}

An Ergonomic Analysis (EA) was made specifically for this study, to know the aspects related to: (i) the constraints associated with work with computers in a real work situation, (ii) the workers main complains related to the work environment, (iii) the main strategies (postural and interaction) adopted by the workers, (iv) the main worker's physical constraints and (v) the work organization and its influence in the problems reported by the workers.

The EA was carried out in a Portuguese service company, from January of 2008 to January of 2009. The company, with more than 15 years in the Portuguese market, had 150 workers. Those 50 worked in the office (management).

During the EA some workstations were select to be filmed and to be used in the video-analysis. The main criteria for selection were: a) with similar workload and functions; b) minimum of six hours on the job; c) good conditions to install the cameras; d) similar workstations; and e) worker with declared good health.

The main tasks reported by the workers who were selected to participate in this study were:

- Task A - Customer Service:

* Talking with others by phone or personally;

* Customer Service (in person or by phone)

Management of customer complaints (or answering e-mails);

* Improve trade relations, enhance the current portfolio of clients (contact with dissatisfied customers, by phone, in order to avoid the end of the contract with the company);

* Charging the customer when it is necessary.

- Task B - Archive and handling paper documents:

* Management of the cancellations of the contracts with clients (procedure involving mostly the filling out of paper forms and and changing data into the computer);

* Make a request form to collect the equipment on the client (end of contract), to confirm the payment and reimburse the customer when necessary;

* Confirm every month, the payment information;

- Task C - Monitoring payments, updating bank accounts, agendas, activities and other events:

* Customer profitability analysis (using spreadsheets, paper and computer);

* Meet schedule established by the administration of the sector (see a list of events and alerts, which are pending in the computer system, and take the necessary decisions for its completion);

* Changing business conditions and customer data (access to medical records of each client in the computer system);

* Correcting the billing in the computer system (when the price change, also change the spreadsheet)

- Task D - Making letters and reports using the computer (typing):

* Fill the maps of customer service (mainly done using the computer); 
* Insert on the computer system the tabs with the payments or customer orders (information from paper invoices);

* Communicate to customers the delivery day;

* Receive, record and answer the customers complaints;

* Clarify customers' questions and answer their suggestions.

All 30 workstations had similar equipment, such as: computer monitor, mouse, telephone and some paper holders. All of them declared to use the computer more than a half of their daily work journey.

\subsection{Phase II-Definition of categories of Interaction}

Generally, the studies assess the upper limbs postures during the work with Visual Display Terminal VDT through the measurement of the angles formed by the segments or through the relation with the predefined anatomical postures (e.g., flexion, extension, abduction). However, few studies were found in the literature which had focused in the measurement of the upper limbs support during a real situation of work with VDT, presenting the distribution of these supports.

Since the identification of the support is easier to observe in video analysis, we chose to record only the behaviors related to the upper limb support, ignoring the positions taken by the upper limbs segments. The categories of this study are divided in: single support, multiple supports and without support. The single support represents the contact of and specific segment with the support surface (e.g., hand on the table). The multiple supports are related to the contact $\mathrm{f}$ two or more body segments with the surface of support (e.g., hand and arm resting on the table). The lack of support is considered when none of the upper limbs are supported. Because the images were collected in real world conditions some situations could be obstructed by some environmental feature. Considering this, a category called "Not visible" was included in the group of Interaction Categories (IC). The IC has the objective of registering the situation where the visualization of the upper limbs is difficult or impossible (e.g., other people in front of the camera obstructing the image).

There were registered fourteen Interaction Categories-ICs, which represents the main means to support the upper limbs during work with VDTs:
(a) Left arm support;
(b) Right arm support;

(c) Left forearm arm support;

(d) Right forearm arm support;

(e) Left elbow support;

(f) Right elbow support;

(g) Left hand support;

(h) Right hand support;

(i) Left upper limbs multiple supports (representing the supports or simultaneous combinations between two or more upper limbs segments, for instance, forearm and hands resting on the table);

(j) Right upper limbs multiple supports (representing the supports or simultaneous combinations between two or more upper limbs segments, for instance, forearm and hands resting on the table)

(k) Without support (Left upper limbs are motion or manipulating objects);

(1) Without support (Right upper limbs are motion or manipulating objects);

(m) Left upper limbs not visible or absent from the video;

(n) Right upper limbs not visible or absent from the video.

In order to avoid that the situations of support were confused with small interactions or contacts with equipment (e.g., keyboard and mouse) it was defined that the support would be characterized, predominantly, by a static behavior ( $>3$ seconds), in which the supported segment is in permanent contact with a surface (e.g., left arm resting on the table).

During the interaction with the keyboard (typing), or using the mouse (navigation), we verify a light contact of the wrist or of the carpal region, with the surface of interaction (the table or the keyboard).

However, due to the constant movement of the hand, wrist and fingers, this kind of support was not considered sufficiently efficient for to bear the weight of the arm. It is also difficult to be identified in the images. In this way, we opted by do not consider the situation in which the hand interacts with the mouse, or the fingers interact with the keys of the keyboard, as a support for the hand.

This did not prevent that other kinds of supports were registered during the interaction with the keyboard and mouse, such as the support of the forearms and of the elbows during the typing and navigation tasks. 


\subsection{Video Record of the User Product-Environment Interaction}

For the image collection in real situation was used a vigilance video system composed of two cameras, the E5582 P\&B and the AEE AR203 color 2.4Ghz, wireless (both with night vision), and both connected to the Sony Multiplexer YS-DX5168 (16 channels). The files were stored using a reader/recorder of DVD, LG, model RH277H. This arrange permitted the continuous recording of eight daily hours.

In order to have the majority of the postures, identified as observation categories, two plans for filming were defined to register the workers sagittal and superior plans.

An observation could be made considering the lateral inclination postures, since these would be better identified with a third camera, located in the frontal plan (front or back). However, the combination of horizontal, superior and sagittal plans is sufficient for identify the inclinations.

Sometimes, some obstructions in the sagittal plan can occur but the superior horizontal plan compensates these difficulties. Like this, the two cameras should be positioned as follow:

Sagittal or lateral plan: a camera is positioned diagonally to the individual's sagittal plan (right or left, according to the orientation of the workstation), and adjusted in way to fit the lower members, upper members, trunk and head for the sitting posture. Whenever possible, to leave a free space of 1.5 meters, approximately, behind the chair's backrest allowing the observation of the individual even with displacements in the workstation.

Superior plan: the camera is positioned in the ceiling, relative to the position of the worker's head, in parallel to his/her transversal plan. The image's frame has to encompass, at least, $90 \%$ of the surface of work, with the worker in the center. The superior plan allows the identification of interactions with objects and twists of the trunk and head. In natural conditions this plan is rarely obstructed, contrarily to what can happen with the sagittal plan. Thus, the superior plan becomes more reliable to the identification of the interactions. Nevertheless, we could verify some restrictions in the identification of the postures of the trunk and lower limbs. The positioning of the camera in the two plans can be seen in the Figure 1

To ensure that the interaction behaviors performed by workers would not be influenced by the characteristics of the used chairs, we chose to standardize this equipment. A specific chair was selected and used by all workers filmed for this study. The selected chair meets all requirements established by the BS EN 1335-1 [10] and ANSI/HFES 100 [11].

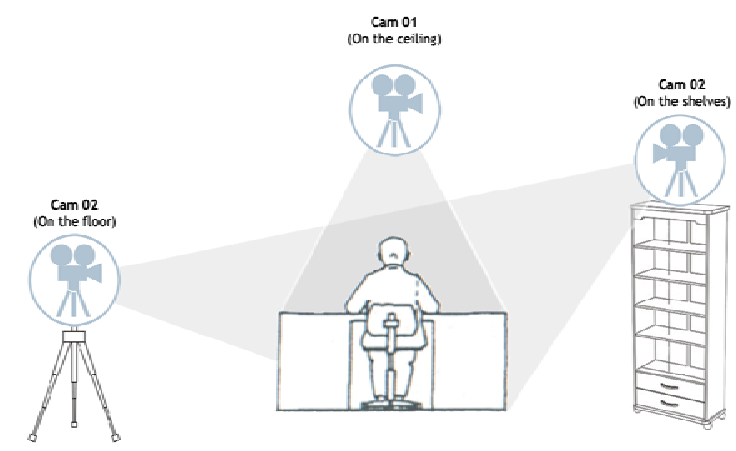

Fig. 1 - The placement of the cameras. The camera 01 was in the ceiling and the camera 02 could be placed in two positions depending on the layout of the workstation.

The cameras remained in each workstation during five days (one week of work), but only the images for three alternate days of work were collected. (e.g., monday, wednesday and friday).

For analysis purposes, there was only considered the first and second days. The third day was used as reserve in case of detection of the following events:

- Technical problems related to the cameras functioning (e.g., errors in the automatic programming of cameras);

- Problems that affected the cameras' performance (e.g., lack of energy);

- Natural problems that affect in the image the viewing conditions of the worker (e.g., obstruction of the viewing plan by external factors such as other worker passing in front of the camera);

- Problems related to the worker's absence from the workstation for periods that were equal or greater than one work shift.

It should be noted that the workers did not know the days when they were being filmed. All clues that could reveal the cameras' operation were hidden (e.g., the lights of the cameras were disabled, and all information panels were covered).

Each day recorded represented 10 hours of images collection (from 8:00 to 18:00), to ensure that even if the worker started his/her activities earlier or finished them later, this period would be registered. However, only eight work hours were analyzed, starting when the worker started his/her activities including lunch time.

The installation and maintenance of equipment were made between 6:00 and 7:00 am, before the workers arrived in order to avoid the contact and the 
influence of the investigator. The investigator met the worker only in the first day of filming to perform an informal interview with the objective of to highlight the study's goal, answering some doubts and to ask the worker to sign a form of consent related to the image collection and confidentiality.

\subsection{Phase IV: Register of the Categories of Interaction}

This study used dynamic events (i.e., video sequence) which allow the investigator to have the notion of continuity of the worker's behavior. This can facilitate the investigator decision about the worker's behavior during the moment of evaluation (e.g., in a situation in which the analysis is done based in a static image, it is impossible for the investigator to know if the image represents a communication process $r$ only a pause in the worker's activity. The same does not happen while using dynamic events).
All video analysis was made based in pre-defined (i.e., five seconds) events. The events were composed by observation categories which represent the worker's activities during the pre-defined period of time. Thus, an event is a group of observation categories which were registered by the investigator during a pre-defined period of time.

The period of time for each event and the interval between each event were configured in software and remained constant throughout the analysis period (Figure 2). Each event is presented to the investigator during five seconds and it is repeated indefinitely until the investigator completes the classification. After configuration, the software automatically controls the video, the intervals, and the events, and also gives internal digital markers in order to identify them. These markers turn the navigation between events easier and allow us to correct previous classifications.

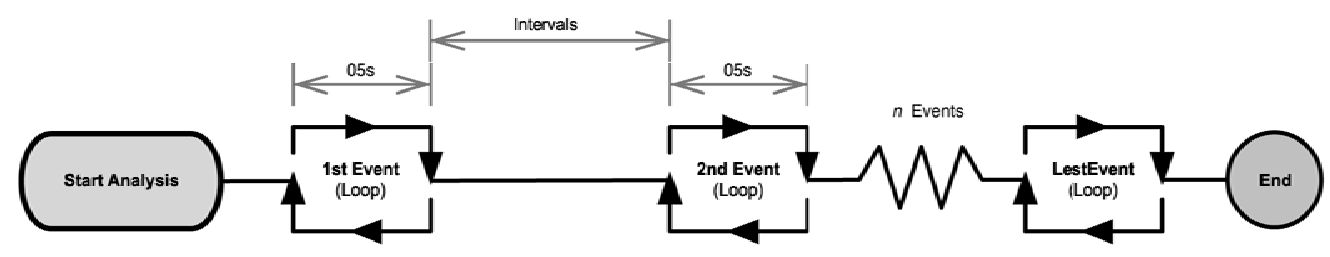

Fig. 2 - Example of an observation. In this example, the observation has " $N$ " events, each one with five seconds of duration and with a fixed interval between them, representing a total of " $Z$ hours" of analysis. The event remains in loop until the investigator completes the classification and proceeds to the next event.

\subsection{Phase V: Data Analysis}

The sample is 46554 events which were extracted from images collected from 30 office workers during two days in real work situations. As mentioned, each event represents five seconds of video with an interval of 25 seconds between them. This represented a total of 388 hours of video. Although over 480 hours of video had been collected, only 388 hours were considered as valid, according to the requirements defined by the method.

The interaction behaviors which were classified in the video images were also registered by a male observer, with 37 years old, and with experience in using observation methods through video-analysis.

The observer has fulfilled all the procedures defined by the method, including regular breaks every 20 minutes of analysis.
In a real situation, the postures for body segments are, generally, combined (e.g., simultaneous twisting and bending of the head). This may raise doubts related to the posture to select while classifying an event. For this cases the observer must to consider the behaviors which were more harmful to the musculoskeletal system, establishing a hierarchy between categories (e.g., situations without support were privileged, even when there was a slight support of one or two seconds per event of five seconds).

For the cases when more than one situation of upper limbs support happened, the observer had to follow some rules:

- Registering the predominant support during the event;

- Registering the harmful support (higher exposure to risk factors). 


\section{Results}

Results were analyzed considering only the ICs for upper limps support. Only the valid register were considered (i.e., all events where the images were obstructed or when the worker was out of his/her workstation were not considered). In this way, only 33800 events of 46554 registered, had the ideal conditions to be analyzed, so this value (33800) will be considered as the total for the valid observations $(100 \%)$ :

- Right arm: arm support 0.06\%; forearm support 31,8\%; elbow support 31,92\%; hand support 7,41\%; multiple supports $12,32 \%$ and without support $16,41 \%$;

- Left arm: arm support 0,11\%; forearm support $53,69 \%$; elbow support $8,70 \%$; hand support 7,97\%; multiple supports $8,43 \%$ e without support $21,11 \%$.

At least one of the upper limbs was supported by the table surface more than $80 \%$ of the registers. However, the distribution of the support is different when right and left bias were considered. This difference may be related to the different needs of interaction with different equipment, such as the keyboard and mouse. The behavior of the right upper limb had a predominance of the forearm and elbow supports $(\sim 64 \%)$. For the left upper limb, the predominance was the support of the forearm $(\sim 54 \%)$. The high occurrence for the elbow support of the right upper limb may be associated with the "V" posture which is adopted during the interaction with the mouse and with the elbow resting on the chair's armrest.

Although the left upper limb has presented more occurrences for the category "without support", the differences between left and right upper limbs were not significant.

\section{Discussion and Conclusion}

According to the video observation, all participants used the mouse with the right hand (their declared dominant hand), in this way, it was expected that the use of the right arm was higher than the use of the left (i.e., increasing the number of occurrences for the right $\mathrm{arm} /$ forearm /hand without support). The "without support" category (which characterizes a dynamic interaction behavior and gesture) had more occurrences for the left upper limb (21.11\%) than for the right. Contrary to the assumption that having the right hand as dominant would provoke more activity of this limb (people use their dominant hand to write, typing and handling objects), results shown that the workers transfer these activities to the left upper limb, which was used for typing, checking notes or lists and handling objects or food. This may be an adaptive reflection derived from the impossibility of using the right limb which was occupied using the mouse.

Since there is no information available considering the same approach and methodology adopted by this study, it was not possible to compare "Ad literam" the results obtained in the case study with the data available in literature.

The results that emerged from this case study are important to turn the studies in Occupational Biomechanics and Physiology fields, related to the real behavior of the upper limbs, more ecological.

In this way, a combination of the systemic and ecological approaches achieved with the adopted method and software with the Biomechanics and Physiology experimental methods will allow the development of more efficient functional requirements for the work with VDTs.

In addition to applications in Ergonomics and Design areas, this method also allows objective assessment of the interaction of the workers with their work devices in the office. It also adds rigor to the epidemiological studies, because it replaces the data obtained through self-reports by data obtained from visible behaviors.

\section{References}

[1] Rebelo, F., E. Filgueiras, and M. Soares (2011), Behavior Video: A Methodology and Tool to Measure Human Behavior; Examples in Product Evaluation, in Human Factors and Ergonomics in Consumer Product Design: Methods and Techniques, W. Karwowski, M.M. Soares, and N.A. S., Editors., CRC Press - Taylor \& Francis. p. 320

[2] Rahmatalla, S., Smith, R., Meusch, J., Xia, T., Marler, T., \& Contratto, M. (2010), A Quasi-static discomfort measure in whole-body vibration. Industrial Health, 48: p. 645-653.

[3] Vieira, E.R. and S. Kumar (2004), Working postures: a literature review. Journal of Occupational Rehabilitation,.

[4] Kumar, S. (2001), Theories of musculoskeletal injury causation. Ergonomics,. 4: p. 17-47.

[5] Sauter, S.L., L.M. Schleifer, and S.J. Knutson (1991), Work posture, work- station design, and musculoskeletal discomfort in a VDT data entry task. Human Factors,. 33: p. 151-167.

[6] IJmker, S., Huysmans, M., Blatter, B. M., Van der Beek, A. J., Van Mechelen, W., \& Bongers, P. M. (2006), Should office workers spend fewer hours at their 
computer? A systematic review of the literature. British Medical Journal.

[7] Spielholz, P., Silverstein, B., Morgan, M., Checkoway, H., \& Kaufman, J. (2001), Comparison of self-report, video observation and direct measurement methods for upper extremity musculoskeletal disorder physical risk factors. Ergonomics,. 44(6): p. 588-613.

[8] David, G.C. (2005), Ergonomic methods for assessing exposure to risk factors for work-related musculoskeletal disorders. Occupational Medicine.

[9] Serranheira, F.M.S. (2007), Lesões MúsculoEsqueleticas Ligadas ao Trabalho: que métodos de avaliação do risco?, in Escola Nascional de Saúde Pública. Universidade Nova de Lisboa: Lisboa. p. 290.

[10] British Standards Institution, Office furniture. Office work chair. Dimensions. Determination of dimensions., in $B S E N$ 1335-1:2000. 2000, British Standards Institution: London, UK.

[11] American National Standards Institute (ANSI), Human Factors Engineering of Computer Workstations as an American National Standard., in ANSI/HFES 100-2007. 2007, Human Factors and Ergonomics Society. 